REGARDS

SUR LECONOMIE ALLEMANDE

BULLETIN ECONOMIQUE DU CIRAC
Regards sur l'économie allemande

Bulletin économique du CIRAC

$85 \mid 2008$

Varia

\title{
La force tranquille
}

Isabelle Bourgeois

\section{OpenEdition}

Journals

Édition électronique

URL : http://journals.openedition.org/rea/413

DOI : 10.4000/rea.413

ISBN : 978-2-8218-0866-9

ISSN : 1965-0787

Éditeur

CIRAC

Édition imprimée

Date de publication : 1 mars 2008

Pagination : 3-4

ISSN : 1156-8992

Référence électronique

Isabelle Bourgeois, "La force tranquille», Regards sur l'économie allemande [En ligne], 85 | mars 2008, mis en ligne le 02 juin 2008, consulté le 15 septembre 2020. URL : http://journals.openedition.org/rea/ 413

(c) CIRAC 


\section{La force tranquille}

L'Allemagne aborde l'année 2008 sur des bases économiques solides. "Les piliers de la croissance sont intacts » malgré l'évolution de la parité $€ / \$$, le ralentissement conjoncturel américain ou le renchérissement des matières premières comme des produits pétroliers, explique la Bundesbank dans son rapport de février. En 2007, la croissance a été de $+2,6 \%$ (en données CVS) comme prévu, révèle Destatis, malgré un léger tassement durant le dernier trimestre. Une fois de plus, l'Allemagne a confirmé son titre de championne du monde à l'export, avec un excédent commercial de 198,8 milliards €, tout en voyant se consolider ses fondamentaux internes sur la base d'une activité industrielle toujours aussi soutenue : investissements et consommation privée.

De ce fait, malgré les incertitudes sur l'évolution conjoncturelle mondiale, l'Allemagne envisage 2008 avec optimisme, comme le révèlent les trois principaux baromètres du climat économique. L'indice ZEW (milieux financiers), s'est redressé en février, confirmant que la crise internationale du crédit bancaire " ne devrait pas tirer la conjoncture allemande dans une spirale descendante » (communiqué du 12-02). Cela concorde avec l'analyse de la Bundesbank pour qui «le système bancaire allemand a dans l'ensemble bien maîtrisé les turbulences des derniers mois » (rapport de février). L'indice ifo (entreprises industrielles) remonte depuis janvier : les entreprises, dont les carnets de commande débordent, jugent favorablement leur situation actuelle, même si elles envisagent l'avenir avec plus de scepticisme, ne sachant trop comment évolueront le marché mondial et le cadre des activités en Allemagne. L'indice GfK (consommateurs), reste stable depuis janvier. Certes, la hausse des prix enregistrée ces derniers mois, notamment dans l'alimentaire, nourrit l'inquiétude des ménages et les amène à hésiter à s'équiper en biens de consommation durable, mais dans le même temps, l'embellie continue sur le marché de l'emploi et la perspective de hausses salariales dans une série de branches dont les conventions collectives expirent cette année leur restituent un peu de confiance en l'avenir. Pour le dire autrement : le moral des ménages ne s'est pas effondré, et leur propension à consommer dépendra majoritairement de l'évolution de l'inflation ressentie.

Malgré les restructurations en cours, le système bancaire allemand a «prouvé sa solidité et sa capacité à assumer son rôle » (BuBa), l'industrie investit pour pouvoir répondre aux commandes qui continuent d'affluer, les activités du BTP se sont stabilisées, et les ménages se sont accommodés du nouveau taux de TVA. Un quatrième baromètre qui mesure, lui, l'état de l'opinion une fois par an et est publié à la fin décembre : celui de l'Institut für Demoskopie d'Allensbach (IfD), révèle que $50 \%$ des Allemands « envisagent 2008 avec espoir ». Depuis 1949, cet 'indice' reflète avec une remarquable fiabilité la tonalité de fond qui marque la société et sous-tend l'orientation des activités (www.demoskopie.de).

Les grands indicateurs conjoncturels incitent à l'optimisme, même si les prévisions de croissance pour 2008 ont été récemment revues à la baisse par plusieurs institutions. Elles ne s'en situent pas moins aux alentours des $+1,6 \%$ estimés par la Commission européenne, compte tenu de la récente dégradation de la conjoncture mondiale comme des incertitudes quant à l'évolution des taux de change ou des prix des matières premières.

La demande mondiale continue en tout cas de remplir les carnets de commande de l'industrie allemande ( $+5 \%$ au dernier trimestre ; ministère fédéral de l'Economie), surtout dans ces biens d'investissement $(+5,6 \%)$ que leur qualité rend relativement insensibles à un euro fort. La bonne tenue des investissements d'équipement $(+7,9 \%$; Destatis) laisse penser que l'industrie produira encore à la limite de ses capacités durant les prochains mois, même si une partie de cette hausse est imputable à l'anticipation des investissements avant la fin d'un régime très favorable des amortissements au $1^{\text {er }}$ janvier. Mais ces effets sont contrebalancés par l'entrée en vigueur à la même date de dispositions incitatives adoptées dans le cadre de la réforme de la fiscalité des entreprises (voir REA 84/07). Et si les investissements en construction ont baissé de $-2,2 \%$ au troisième trimestre de 2007, cela est dû avant tout à l'habitat (-3,9\%).

Sur l'ensemble de 2007, l'activité industrielle a crû de $+5,2 \%$ (Destatis) ; suivie par les services aux entreprises $(+3,1 \%)$, la catégorie transport, commerce et hôtellerie $(+2,3 \%)$ et même l'agriculture, la sylviculture et la pêche $(+2,7 \%)$, de même que le BTP $(+1,7 \%)$. La consommation, en revanche, a stagné $(-0,2 \%)$, celle des ménages ayant reculé de $-0,3 \%$. Pourtant, leur revenu disponible s'est accru de $+1,6 \%$. Mais leur marge de manœuvre a été absorbée par une hausse de la fiscalité indirecte et des prix administrés - autant d'effets particuliers expliquant un taux d'inflation proche des $3 \%$ (BuBa). Quant au taux d'épargne, il est monté à 10,8 \% dans un contexte d'incertitudes sur l'avenir des retraites.
Indices ZEW, ifo et Gfk : une tendance de fond à la confiance

Baromètre IfD : $50 \%$ des Allemands abordent 2008 " avec espoir »

Une croissance de $+1,6 \%$ pour 2008

L'investissement orienté à la hausse

Hausse de l'activité industrielle en 2007 
Emploi en hausse budget équilibré

L'objectif 2008 du gouvernement : " Garder le cap "...

... souffre quelques exceptions dans le contexte électoral actuel
Ce qui soutient l'optimisme des Allemands, c'est la nette embellie sur le marché de l'emploi et la réduction du déficit à zéro. Non seulement, le nombre d'actifs occupés a augmenté de 650000 (+1,7\%), notamment dans l'industrie qui a embauché 85000 personnes, mais le chômage a poursuivi sa décrue (623 000 chômeurs de moins), ce qui abaisse le taux de chômage (critères OIT) à 7,9 \% à la fin 2007, comme le révèle la BuBa qui en profite pour rappeller que, pour que l'embellie continue, la politique salariale des partenaires sociaux doit «garder la mesure » et veiller à « une structure flexible » de l'échelle des salaires. Quant au budget allemand, il est excédentaire en 2007, certes de très peu (200 millions € ; Destatis), mais il sort du rouge pour la première fois depuis 1989.

Si on fait abstraction des incertitudes qui planent sur la conjoncture mondiale, l'Allemagne récolte aujourd'hui le dividende des réformes engagées sous le précédent gouvernement fédéral, ainsi que le souligne le programme 2008 du gouvernement fédéral, résumé dans le dernier rapport mensuel du ministère fédéral de l'Economie (daté de mars). En réponse aux préconisations du Conseil des Sages dont le rapport annuel s'intitulait « Ne pas remettre en jeu l'acquis ! » (voir REA 84/07), le gouvernement affirme « prendre au sérieux les craintes » des Sages et a rassemblé sous l'intitulé « Garder le cap » ses objectifs politiques pour 2008. Sa priorité reste la lutte contre le chômage, notamment via la compression durable des cotisations sociales à moins de $40 \%$ du salaire brut. Et de fait, c'était là l'un des deux objectifs de la hausse de la TVA (l'autre étant la hausse de l'encours fiscal pour consolider le budget) : la mesure annoncée (abaissement de la cotisation chômage à $3,3 \%$ du salaire) est entrée en vigueur le $1^{\mathrm{er}}$ janvier. Le gouvernement salue également toutes les mesures de flexibilisation qui ont contribué, ces dernières années, à l'embellie de l'emploi : temps partiel, CDD, intérim, minijobs, et - apport des partenaires sociaux -, clauses d'ouverture et modulation du temps de travail.

Peu après la publication de ce rapport, le ministre, Michael Glos (CSU), saisit l'occasion de la révision des prévisions de croissance du FMI (à $+1,5 \%)$, assorties de la recommandation de ne pas faire machine arrière sur les réformes, pour abonder dans le même sens: « Je me sens pleinement conforté dans mon scepticisme envers l'instauration de SMIC légaux » (communiqué du 22-02). Et pourtant, le gouvernement fédéral avait fait voter, à la fin 2007, un tel SMIC dans le secteur postal (voir dans ce numéro), qui réintroduit une nouvelle rigidité dans des activités qui offraient justement ces emplois « flexibles » permettant d'absorber une main d'œuvre peu qualifiée. Mais c'était pour satisfaire le partenaire de coalition, en la personne d'Olaf Scholz (SPD), ministre fédéral du Travail et vice-chancelier, et dans le contexte des élections parlementaires en Basse-Saxe, Hesse et à Hambourg.

LA BONNE TENUE DES FONDAMENTAUX ECONOMIQUES A UN REVERS : elle semble autoriser le relâchement dans l'effort de réformes et favorise l'émergence de fortes tentations redistributives. La hausse généralisée des salaires réclamée par le syndicat ver.di dans le secteur des services via l'extension du SMIC postal à d'autres branches, la revendication d'une forte hausse dans la fonction publique, l'augmentation disproportionnée (+5,2\%) conclue en février dans la sidérurgie, l'arrivée prochaine à échéance d'un certain nombre de conventions dans des branches clefs de l'industrie (dont la métallurgie), comportent un risque indéniable pour la santé d'une économie allemande qui sort tout juste de convalescence.

II pousse les acteurs économiques à rappeler le gouvernement de coalition à ses 'devoirs'. Ainsi Werner Schnappauf, le nouveau président de la Fédération de l'industrie (BDI): « L'industrie génère à elle seule plus de la moitié de la croissance, ce qui lui donne le droit légitime d'exiger que soient données des conditions-cadre permettant à la croissance de se développer » (FAZ.NET, 15-02). Et de réclamer une politique de hausse des qualifications plutôt que l'instauration de salaires minima légaux. Wolfgang Franz, patron de l'institut ZEW, rappelle, au vu de la hausse de $+2,5 \%$ en moyenne des salaires conventionnels en 2007, que c'est la modération salariale de la décennie écoulée qui a largement contribué à l'embellie sur le marché de l'emploi, recommandant aux syndicats « de ne pas mettre maintenant en danger ce succès - dans l'intérêt des salariés » (Welt Online, 19-01). Quant à Axel Weber, président de la Bundesbank, il met en garde contre la tentation d'un deuxième tour. «La politique salariale comme la politique budgétaire ne doivent pas perdre la mesure ; elles aussi ont la responsabilité de veiller à la stabilité des prix ». Et de poursuivre : «C'est à raison que la chancelière a récemment évoqué l'inflation comme 'la forme la plus perfide qui soit de l'expropriation des petits épargnants sans patrimoine' » (Das Parlament, 11-02).

Les risques qui pèsent sur l'économie allemande en 2008 ne sont pas tant monétaires ni ne se posent en termes de compétitivité industrielle - les risques résident dans un climat de surenchère sociale aux accents populistes déclenchée par la longue série d'échéances électorales de ce début 2008 jusqu'aux élections au Bundestag à l'automne 2009.

Isabelle Bourgeois (28-02-2008) 